\title{
Tobacco craving and withdrawal symptoms in psychiatric patients during a motivational enhancement intervention based on a 26-hour smoking abstinence period
}

\author{
Ineke Keizer1,2, Marianne Gex-Fabry', Patrice Croquette', Jean-Paul Humair ${ }^{2,3}$, Aqal N. Khan ${ }^{1}$
}

\begin{abstract}
INTRODUCTION In psychiatric patients, tobacco withdrawal symptoms are frequently seen as a barrier to smoking cessation; however, further studies are warranted in this specific population.

METHOdS Patients receiving in- or out-patient mental health care participated in a motivational enhancement program including a 26-hour tobacco abstinence experience with professional support and optional nicotine replacement therapy. The study included 174 subjects, of whom 159 were evaluated 1 week before and at the end of the 26-hour abstinence period. Repeated assessments included the Minnesota Nicotine Tobacco Withdrawal Scale Revised (MNWS-R), two items of the Mood and Physical Symptoms Scale (MPSS) regarding craving, the State-Trait Anxiety Inventory (STAI-S), the Beck Depression Inventory (BDI-21), and the World Health Organization Well-Being Index (WHO-5).

RESULTS More than half the participants (52.3\%) succeeded in 26-hour smoking abstinence. Craving was the most frequent MNWS-R withdrawal symptom (28.3\% scored $\geq 3$ on a $0-4$ scale). Comparison of pre- and post-intervention data revealed significant improvements in 13 of 16 MNWS-R symptoms as well as craving (MPSS) and well-being, and significant decreases in anxiety and depression. Increasing MNWS-R craving scores and greater depression were both significantly associated with lower success in the 26-hour smoking abstinence period.

concLusions The negative effects of tobacco withdrawal symptoms in psychiatric patients may be substantially overestimated. Participation in a supportive structured motivational intervention with a 26-hour smoking cessation experience was well tolerated and contributed to temporary improvements in mental state. Craving is an interesting symptom to evaluate during smoking cessation attempts.
\end{abstract}

\section{INTRODUCTION}

Among addictions, smoking is the strongest and most difficult to stop, as demonstrated by cessation rates in randomized trials with placebo or no-treatment controls. Indeed, success rates at 6 months have been reported to be $47 \%$ for cocaine, $44 \%$ for opioids, $18 \%$ for alcohol, and only $8 \%$ for nicotine ${ }^{1}$. According to many smokers, craving is the major reason for failing or not attempting to stop. This concept has been described as 'an abnormal desire or need to take a drug $^{2}$, including conscious and unconscious aspects. Although a large consensus supports the importance of craving, this construct remains complex to define and explain ${ }^{3-5}$. Craving is seen as a core symptom of addiction but also appears as a withdrawal effect in cessation. Craving is thus present in both smokers who repeatedly smoke another cigarette and smokers abstaining from smoking during a cessation attempt. In both cases, craving results from nicotine/tobacco deprivation, increasing shortly after abstinence 
(within hours or less) and lasting for several weeks or longer ${ }^{6,7}$. Craving during regular smoking or smoking cessation does not appear qualitatively different, but, during a cessation attempt with a longer abstinence period, discomfort increases, and a larger set of withdrawal symptoms occur together with the urge to smoke. Diagnostic criteria for nicotine withdrawal, according to DSM-IV ${ }^{8}$, include: irritability, frustration or anger; anxiety; difficulty concentrating; restlessness; decreased heart rate; increased appetite or weight gain; dysphoric or depressed mood; and insomnia. Controversy regarding whether craving should be considered a withdrawal symptom is illustrated by its inclusion in the criteria for nicotine dependence in the DSM-III edition, absence in the DSM-IV, and reintroduction in the DSM-V 'tobacco use disorder' entry. The different withdrawal symptoms are highly correlated, and factor analyses have suggested using a total score ${ }^{9}$, although combining elements specific to smoking (craving) with those less specific (e.g. anxiety, depression, irritability), or combining 'psychological' variables with somatic or physiological variables (hunger) may warrant further consideration $^{10,11}$.

The high prevalence of smoking in psychiatric patients is well established, the odds that a patient with bipolar disorder will become a current smoker are 3.5 times higher than those from people from the worldwide general population ${ }^{12}$, and are even higher for patients with schizophrenia. Rates of $36.1 \%^{13}, 53 \%^{14}$ or $67 \%^{15}$ are described in patients with schizophrenia or non-affective psychosis. Although this population accounts for a growing proportion of all smokers ${ }^{13,16}$, barriers prevent optimal interventions ${ }^{17,18}$. The assumption that smoking cessation may be a risk factor for increased psychopathology in psychiatric patients is widespread ${ }^{19,20}$, although most recent studies emphasize the benefits of cessation ${ }^{21}$ and the selfmedication hypothesis of smoking is questioned ${ }^{22}$. In our clinical practice, we frequently encounter patients reporting that they are unable to stop smoking because they fear being unable to tolerate craving symptoms, or missing smoking's benefits of anxiety or stress relief. Evaluation of nicotine withdrawal symptoms in psychiatric patients has encountered methodological difficulties, owing to the overlap of these symptoms with those common in several mental health disorders (e.g. anxiety, depression, and sleep disturbance $)^{23}$. In a sample of smokers with attention-deficit hyperactivity disorder (ADHD) assessed before any cessation attempt, nicotine withdrawal symptoms (difficulty concentrating, restlessness or impatience, and anxiety or nervousness) positively correlated with current ADHD symptoms. These symptoms were significantly more severe in smokers with a history of ADHD than in smokers without ADHD, thus illustrating the difficulty in interpreting symptoms potentially influenced by both mental disorders and tobacco withdrawal ${ }^{24,25}$. Psychiatric patients have frequently been excluded from research on smoking cessation and development of instruments in this domain, thus calling into question the generalizability of results to populations with mental health conditions. Therefore, careful documentation of nicotine deprivation symptoms in relation to psychiatric symptoms remains a challenge in these populations, particularly regarding tobacco use and cessation.

The present study investigated nicotine withdrawal symptoms in a sample of smokers presenting with psychiatric disorders and receiving mental health care from in- or out-patient public services. They participated in a short motivational enhancement program based on a 26-hour smoking abstinence period. The first aim was to measure the prevalence of withdrawal symptoms and to study changes before and during the intervention in this specific population, by using the Minnesota Nicotine Withdrawal Scale Revised (MNWS-R or MTWS-R) ${ }^{26,27}$. The second aim was to analyze the factor structure of the MNWS-R and correlations with specific instruments measuring anxiety, depression, and craving. The third aim was to investigate aggravation or improvement in withdrawal symptoms during short-term abstinence and their association with successful or unsuccessful smoking abstinence.

\section{METHODS}

\section{Setting and intervention}

Participants were patients receiving in- and outpatient services at a public psychiatric institution, the Department of Mental Health and Psychiatry of the Geneva University Hospitals. The in-patient 
setting comprised seven acute care or rehabilitation units, and the out-patient setting comprised four ambulatory sectors providing intensive day care and consultation services. An indoor smoking ban applied to all settings, but patients whose condition allowed them to go outdoors were able to smoke there without restrictions. An intervention was designed to motivate smoking cessation in psychiatric patients. This multicomponent intervention (called 'Journée parenthèse' or 'Day off') began on Thursday 8:30 am and ended on Friday 10:30 am, and was the same for in- and out-patients. It was based on a 26hour tobacco abstinence period and a structured day program during which participants benefitted from group support and attended thermal baths, restaurants, and tobacco information sessions, and participated in entertaining activities. Nicotine replacement therapy (NRT) was optional but strongly recommended. The aim of the program was to induce a strong positive experience and personal involvement, in order to mobilize motivational resources initiating a smoke-cessation process, according to the theory that positive outcome experiences influence motivational and intention forming elements, which may lead to behavioral changes ${ }^{28}$. The intervention can be considered successful when participants have experienced a positive outcome related to smoking during the 'Day off' intervention. A detailed description of this motivational enhancement program has been published elsewhere ${ }^{29}$.

\section{Recruitment}

Staff from all units of the institution received information about the intervention. Patients were contacted by their care providers and invited to participate. Subjects could be enrolled if they had smoked recently (regardless of frequency of consumption) and if their psychiatric symptoms and clinical state were stable enough to integrate group participation and multiple activities. Further, they were required to be willing to participate and to commit to abstain from smoking during 26 hours. Repeated participation was possible, but only data related to the first enrolment were used in this study. The exclusion criteria were cognitive impairment or insufficient French fluency. The study protocol was approved by the ethics committee of the Geneva University Hospitals. All participants provided written informed consent before entering the study.

Among 389 participants enrolled in the program over a 7-year period, 174 were included in the study (115 inpatients and 59 outpatients). The reasons for non-inclusion were cognitive impairment, language barriers, or lack of informed consent $(n=116)$; repeated participation in the program $(n=48)$, absence on the intervention day, e.g. because of worsened clinical condition or discharge from the hospital $(n=43)$; or leaving the program during the first hours $(n=4)$. Four other participants were excluded because they had recently stopped smoking but participated to reinforce their motivation. Among 174 subjects in the study, 159 were reassessed after the 26-hour intervention. Fifteen patients were not reassessed for various reasons (staying asleep, having another priority appointment, and lack of motivation, possibly in relation to resumed smoking).

\section{Study design and instruments}

Participants were evaluated on two occasions. The first interview took place during the week preceding the intervention: sociodemographic and diagnostic information was collected, and psychological and tobacco-related instruments were used, as described below. The second evaluation was performed with the same instruments, after the intervention and 26-hour smoking abstinence period (Friday morning).

Withdrawal symptoms were measured with the MNWS-R/MTWS-R scale ${ }^{26,27,30}$, which includes 16 items (anger or frustration, anxiety, depression, craving, difficulty concentrating, increased appetite, insomnia, restlessness, impatience, constipation, dizziness, coughing, dreaming or nightmares, nausea, sore throat, and hot flushes or shivers) rated on a 4 -point Likert scale $(0=$ not present to $3=$ severe). Craving was also measured with the total score of two items of the Mood and Physical Symptoms Scale ${ }^{31}$ (MPSS): 'How much of the time have you felt the urge to smoke today?' and 'How strong have the urges been today?' (from $0=$ not at all/no urges to $5=$ all the time/extremely strong). MNWS-R and MPSS were proposed at preevaluation for collection of baseline data, even if patients were smoking 'as usual'. The Heaviness of Smoking Index ${ }^{32,33}$ (HSI) was calculated on the basis of two questions of the Fagerström Test for Nicotine Dependence (FTND): 'How many cigarettes a day 
do you smoke?' (answers in four categories) and 'How soon after you wake up do you smoke?' (four categories). Readiness to stop smoking was evaluated with Biener's Contemplation Ladder ${ }^{34}$. Anxiety and depression were measured with the State-Trait Anxiety Inventory, state part ${ }^{35}$ (STAI-S) and the Beck Depression Inventory ${ }^{36}$ (BDI-21), respectively. Well-being was evaluated with the WHO-5 index ${ }^{37,38}$, with raw scores with a cut-off point at 11 used in psychiatric settings, to assess well-being ${ }^{39}$. Exhaled carbon monoxide ( $\mathrm{CO})$ was measured with a piCO+ Smokerlyzer (Bedfont, UK) at the start and end of the first intervention day (Thursday 9:15 am and 6:15 pm) and on the next morning (Friday 10:15 am).

\section{Statistics}

Categorical variables were described with frequency tables ( $\mathrm{n}, \%)$. Ordinal and continuous variables were described with medians (ranges). Differences between independent groups were tested with Fisher's exact test for categorical variables and the Mann-Whitney U-test for ordinal and continuous variables. Change over time was tested with the nonparametric sign test (two time points) or the Friedman analysis of variance (more than two time points). Spearman's rank correlation coefficient $(\rho)$ was used to test associations between dimensions. The internal structure of the 16-item MNWS-R was explored with principal component analysis with oblique rotation (promax method) to allow for possible correlations between components. The number of retained components was determined according to the following criteria: 1) Kaiser's criterion, i.e. retaining components with eigenvalues $\geq 1 ; 2$ ) the scree plot method, i.e. retaining components in the steep part of the graph; and 3) percentage explained variance. Items with loadings of at least 0.50 were considered to be part of a given factor, provided that there was no overlap with other factors (cross-loadings $<0.50$ ). Statistics were computed in SPSS version 22 (IBM Corporation, Armonk, NY). All tests were two-tailed, with the significance threshold at 0.05 .

\section{RESULTS}

\section{Sociodemographic, clinical, and smoking characteristics at pre-intervention}

Characteristics of the study sample are summarized in Table 1 . The most frequent primary diagnoses were mood disorders $(40.2 \%)$ and psychotic disorders (41.4\%). According to the anxiety (STAI-S) and depression rating scales (BDI-21), 55.0\% had moderate to very high anxiety scores, and $40.5 \%$ had moderate or severe depression scores.

One-third were heavy smokers ( $>20$ cigarettes/ day), and the median $\mathrm{CO}$ was $21 \mathrm{ppm}$ (range 1-100). More than half $(51.7 \%)$ were in the precontemplation stage, and $75.7 \%$ had not made

Table 1. Sociodemographic, clinical and smoking characteristics at pre-intervention ( $\mathrm{N}=174)$

\begin{tabular}{|c|c|c|c|}
\hline & & n & $\%$ \\
\hline \multicolumn{4}{|l|}{ Sociodemographic characteristics } \\
\hline Gender & Male & 100 & 57.5 \\
\hline Age [median, min-max (range)] & & 35.5 & $17-64(47)$ \\
\hline \multirow[t]{3}{*}{ Education $(\mathrm{N}=170)$} & Compulsory or less & 69 & 40.6 \\
\hline & Intermediate & 87 & 51.2 \\
\hline & Higher & 14 & 8.2 \\
\hline \multirow[t]{2}{*}{ Financial condition $(\mathrm{N}=169)$} & Disability pension or social aid & 107 & 63.3 \\
\hline & Worker or student (no long-lasting disability) & 62 & 36.7 \\
\hline \multicolumn{4}{|l|}{ Clinical characteristics } \\
\hline \multirow[t]{4}{*}{ Primary diagnosis } & Psychoactive substance disorders & 10 & 5.7 \\
\hline & Psychotic disorders & 72 & 41.4 \\
\hline & Mood disorders & 70 & 40.2 \\
\hline & Other disorders & 22 & 12.6 \\
\hline \multirow[t]{3}{*}{ Comorbidity (ICD-10 psychiatric diagnoses) } & 1 diagnosis & 79 & 45.4 \\
\hline & 2 diagnoses & 56 & 32.2 \\
\hline & 3 or more diagnoses & 39 & 22.4 \\
\hline
\end{tabular}


Table 1. Continued

\begin{tabular}{|c|c|c|c|}
\hline \multirow{2}{*}{ Clinical characteristics } & & \multirow[t]{2}{*}{ n } & \multirow[t]{2}{*}{$\%$} \\
\hline & & & \\
\hline \multirow[t]{5}{*}{ Anxiety (STAI-S) $(\mathrm{N}=151)$} & Very low $(<36)$ & 36 & 23.8 \\
\hline & Low (36-45) & 32 & 21.2 \\
\hline & Medium (46-55) & 46 & 30.5 \\
\hline & High (56-65) & 26 & 17.2 \\
\hline & Very high (>65) & 11 & 7.3 \\
\hline \multirow[t]{4}{*}{ Depression (BDI-21) $(\mathrm{N}=153)$} & Absent $(<10)$ & 33 & 21.6 \\
\hline & Mild (10-19) & 58 & 37.9 \\
\hline & Moderate (20-29) & 41 & 26.8 \\
\hline & Severe $(\geq 30)$ & 21 & 13.7 \\
\hline \multirow[t]{2}{*}{ Well-being $(\mathrm{WHO}-5)(\mathrm{N}=163)$} & Positive well-being ( $\geq 11$ ) & 96 & 58.9 \\
\hline & Low well-being $(<11)$ & 67 & 41.1 \\
\hline \multicolumn{4}{|l|}{ Smoking characteristics } \\
\hline \multirow[t]{4}{*}{ Cigarettes per day $(\mathrm{N}=173)$} & $\leq 10$ & 56 & 32.4 \\
\hline & $11-20$ & 59 & 34.1 \\
\hline & $21-30$ & 38 & 22.0 \\
\hline & $\geq 31$ & 20 & 11.6 \\
\hline $\begin{array}{l}\text { Heaviness of smoking index (HSI) [median, min-max } \\
\text { (range)] }(\mathrm{N}=171)\end{array}$ & & 4.0 & $1-7(6)$ \\
\hline Carbon monoxide [median, min-max (range)] (N=171) & ppm & 21.0 & $1-100(99)$ \\
\hline \multirow[t]{2}{*}{ Quit attempt in past 6 months $(\mathrm{N}=169)$} & Yes & 41 & 24.3 \\
\hline & No & 128 & 75.7 \\
\hline \multirow[t]{2}{*}{ Stage of change } & Pre-contemplation & 90 & 51.7 \\
\hline & Other stage & 84 & 48.3 \\
\hline $\begin{array}{l}\text { Preparation to quit (Biener) [median, min-max (range)] } \\
(\mathrm{N}=173)\end{array}$ & & 7.0 & $0-10(10)$ \\
\hline $\begin{array}{l}\text { Craving (MPSS subscale) [median, min-max (range)] } \\
(\mathrm{N}=173)\end{array}$ & & 5.0 & $0-10(10)$ \\
\hline
\end{tabular}

a cessation attempt in the previous 6 months. The nicotine dependence (HSI score) did not significantly correlate with anxiety $(\rho=0.05, \mathrm{p}=0.56)$ or depression $(\rho=0.02, p=0.85)$, but was significantly associated with decreased well-being (WHO-5; $\rho=-$ $0.15, p=0.05$ ) and increased craving (MPSS; $\rho=0.47$, $\mathrm{p}<0.001)$. The HSI score was not significantly different for NRT users/no-users ( $\mathrm{n}=130$, median $=4$ vs $\mathrm{n}=19$, median $=4$; Mann-Whitney U-test $\mathrm{p}=0.10$ ). The number of cigarettes smoked per day might have been related to NRT use (medians of 20 for users and 10 for the no-NRT subgroup; Mann-Whitney U-test $\mathrm{p}=0.05$ ).

Comparison of in- and out-patients showed no significant differences for any diagnostic or smoking characteristics; these groups differed only in age (inpatients were younger; Mann-Whitney U-test $\mathrm{p}<0.001$ ) and sex (inpatients included more males; Fisher's exact test $\mathrm{p}=0.04$ ) but not in other sociodemographic data.

In Table 1, some data are missing because of non-reliable answers (i.e. financial condition or past cessation attempts) but mainly because of concentration difficulties related to mental health conditions when longer questionnaires were used (i.e. STAI anxiety or BDI depression).

\section{Outcomes of the 26-hour smoking abstinence intervention}

NRT was largely used during the 'Day off' intervention: $86.8 \%$ of participants took fast and/or slow acting products, and the dosage was adapted to the usual cigarette consumption according to standard medical recommendations. $\mathrm{CO}$ decreased significantly during the intervention (median $18 \mathrm{ppm}$ at 9:15 at the start of the intervention; $7 \mathrm{ppm}$ at 18:15 at 
the end of the first day; and $6 \mathrm{ppm}$ at 10:15 on the next day; Friedman test $\mathrm{p}<0.001)$. At the end of the intervention, the median $\mathrm{CO}$ was $4 \mathrm{ppm}$ for those succeeding in abstinence and $11 \mathrm{ppm}$ for those not succeeding.

Of 174 participants, $8.6 \%(n=15)$ smoked one or several cigarettes during the intervention day, and 9 out of $10(89.7 \%, \mathrm{n}=156)$ did not smoke during the intervention day (9-hour abstinence). Smoking abstinence was further maintained during the 26hour period after participants spent the evening and night in an uncontrolled environment (the usual living place for outpatients or a hospital ward for inpatients $)$ in $52.3 \%(n=91)$ of participants. Information was missing for three participants (e.g. $\mathrm{CO}$ value inconsistent with self-reported data). Participants who did and did not succeed in 26hour abstinence did not differ in baseline anxiety, depression, well-being, and craving, but nicotine dependence (HSI) was significantly lower among those who succeeded (median 4.0 vs 5.0, MannWhitney U-test $\mathrm{p}=0.03$ ).

\section{Withdrawal symptoms during the 26-hour abstinence intervention}

Table 2 shows the percentages of participants with symptoms either absent (score 0 ) or marked/severe (scores 3 or 4) according to the MNWS-R, as reported after the 26-hour abstinence intervention. Craving was the most frequent symptom ( $28.8 \%$ of participants with scores 3 or 4 ), whereas 15 to $22 \%$ reported elevated levels of anxiety, depressed mood, difficulty concentrating, increased appetite, restlessness, and impatience. Five withdrawal symptoms (constipation, dizziness, nausea, sore throat, hot flushes, or shivers) were absent in more than $50 \%$ of subjects. Sleep difficulties and nightmares were present in approximately half of the subjects and were marked in $20-24 \%$ of the subjects. Median scores are provided in Table 3a.

Significant correlations were observed between the MNWS-R item 'anxious, nervous' and the STAI-S anxiety score $(\rho=0.55, p<0.001)$; the item 'depressed mood, sad' and the BDI-2 1 depression score $(\rho=0.45$, $\mathrm{p}<0.001)$; and the item 'desire or craving to smoke' and the MPSS craving score $(\rho=0.69, p<0.001)$.

Further inspection of MNWS-R items according to use of NRT resulted in very small subgroups, thus limiting the possibility and power of statistical analyses. In Table 2, only the item 'craving' had sufficient data for minimal analysis (expected cell counts $>5$ ) and showed no significant differences according to use of NRT and the item craving absent vs marked or severe $(\mathrm{n}=77$; Fisher's exact test $\mathrm{p}=0.38)$.

Table 2. Results on the Minnesota Withdrawal Scale (MNWS-R) during abstinence trial $(\mathrm{N}=152)$

\begin{tabular}{|c|c|c|c|c|c|}
\hline \multirow[t]{2}{*}{ MNUS- $R$ items } & \multirow{2}{*}{$\begin{array}{c}\text { Absenta } \\
\text { n ( }(\%)\end{array}$} & \multirow{2}{*}{$\begin{array}{c}\text { Varked or severeb } \\
\text { n ( (\%) }\end{array}$} & \multicolumn{3}{|c|}{ PCA factor loadingsc } \\
\hline & & & Factor 1 & Factor 2 & Factor 3 \\
\hline Angry, irritable, frustrated & $68(44.7)$ & $19(12.5)$ & 0.79 & 0.27 & 0.38 \\
\hline Anxious, nervous & $40(26.3)$ & $25(16.4)$ & 0.80 & 0.48 & 0.15 \\
\hline Depressed mood, sad & $59(38.8)$ & $26(17.1)$ & 0.66 & 0.49 & 0.19 \\
\hline Desire or craving to smoke & $34(22.4)$ & $43(28.3)$ & 0.68 & 0.27 & 0.18 \\
\hline Difficulty concentrating & $50(32.9)$ & $22(14.4)$ & 0.47 & 0.67 & 0.19 \\
\hline Increased appetite, hungry, weight gain & $57(37.5)$ & $30(19.7)$ & 0.20 & 0.35 & 0.55 \\
\hline Insomnia, sleep problems, awakening at night & $75(49.3)$ & $34(22.4)$ & 0.34 & 0.63 & 0.30 \\
\hline Restless & $52(34.2)$ & $25(16.5)$ & 0.84 & 0.45 & 0.28 \\
\hline Impatient & $39(25.7)$ & $32(21.1)$ & 0.75 & 0.46 & 0.16 \\
\hline Constipation & $105(67.3)$ & $16(10.3)$ & 0.24 & 0.63 & 0.17 \\
\hline Dizziness & $103(67.8)$ & $16(6.6)$ & 0.44 & 0.78 & 0.29 \\
\hline Coughing & $75(49.3)$ & $18(11.9)$ & 0.29 & 0.14 & 0.78 \\
\hline Dreaming or nightmares & $76(50.0)$ & $31(20.4)$ & 0.22 & 0.41 & 0.45 \\
\hline Nausea & $115(75.7)$ & $5(3.3)$ & 0.15 & 0.38 & 0.67 \\
\hline Sore throat & $99(65.1)$ & $12(7.9)$ & 0.22 & 0.21 & 0.80 \\
\hline Hot flushes, shivers & 91 (59.9) & $19(12.5)$ & 0.40 & 0.74 & 0.31 \\
\hline
\end{tabular}

a score $=0$. b score $=3$ or 4 . c bold: loadings $>0.50$. 
Table 3a. Withdrawal symptoms before the intervention and after 26 -hour abstinence trial $(\mathrm{N}=174)$

\begin{tabular}{|c|c|c|c|c|c|c|c|}
\hline & \multirow{2}{*}{$\begin{array}{c}\text { Pre } \\
\text { intervention } \\
\text { Median }\end{array}$} & \multirow{2}{*}{$\begin{array}{l}\text { End of } \\
\text { intervention } \\
\text { Vedian }\end{array}$} & \multicolumn{2}{|c|}{ Improved } & \multicolumn{2}{|c|}{ Worsened" } & \multirow{2}{*}{$\begin{array}{l}\text { Sign } \\
\text { test } \\
\text { p }\end{array}$} \\
\hline & & & $n^{c}$ & $\%$ & $n^{c}$ & $\%$ & \\
\hline \multicolumn{8}{|l|}{ Withdrawal symptoms (MNWS-R) (range 0-4) } \\
\hline Angry, irritable, frustrated $(n=153)^{c}$ & 1.0 & 1.0 & 65 & 42.5 & 25 & 16.3 & $<0.001$ \\
\hline Anxious, nervous ( $n=153)$ & 2.0 & 1.0 & 96 & 62.7 & 12 & 7.8 & $<0.001$ \\
\hline Depressed mood, sad $(n=151)$ & 2.0 & 1.0 & 76 & 50.3 & 23 & 15.2 & $<0.001$ \\
\hline Desire or craving to smoke $(n=152)$ & 2.0 & 2.0 & 70 & 46.1 & 32 & 21.1 & $<0.001$ \\
\hline Difficulty concentrating $(n=153)$ & 2.0 & 1.0 & 77 & 50.3 & 24 & 15.7 & $<0.001$ \\
\hline Increased appetite, hungry, weight gain $(n=151)$ & 1.0 & 1.0 & 49 & 32.5 & 48 & 31.8 & 1.0 \\
\hline Insomnia, sleep problems, awakening at night $(n=153)$ & 1.5 & 1.0 & 67 & 43.8 & 29 & 19.0 & $<0.001$ \\
\hline Restless ( $n=153)$ & 2.0 & 1.0 & 72 & 47.1 & 31 & 20.3 & $<0.001$ \\
\hline Impatient $(n=153)$ & 2.0 & 1.0 & 69 & 45.1 & 37 & 24.2 & 0.003 \\
\hline Constipation $(n=163)$ & 0.0 & 0.0 & 27 & 16.6 & 20 & 12.3 & 0.38 \\
\hline Dizziness ( $n=153)$ & 1.0 & 0.0 & 56 & 36.6 & 23 & 15.0 & $<0.001$ \\
\hline Coughing $(n=152)$ & 1.0 & 1.0 & 53 & 34.9 & 28 & 18.4 & 0.008 \\
\hline Dreaming or nightmares $(n=152)$ & 1.0 & 1.0 & 64 & 42.1 & 27 & 17.8 & $<0.001$ \\
\hline Nausea $(n=153)$ & 0.0 & 0.0 & 35 & 22.9 & 22 & 14.4 & 0.11 \\
\hline Sore throat $(n=152)$ & 0.0 & 0.0 & 37 & 24.3 & 27 & 17.8 & 0.26 \\
\hline Hot flushes, shivers $(n=152)$ & 1.0 & 0.0 & 57 & 37.5 & 22 & 14.5 & $<0.001$ \\
\hline \multicolumn{8}{|l|}{ Craving } \\
\hline MPSS (observed range $0-10)(n=157)$ & 5.0 & 4.0 & 88 & 56.1 & 32 & 20.4 & $<0.001$ \\
\hline \multicolumn{8}{|l|}{ Anxiety } \\
\hline STAI-S (observed range $20-77)(n=120)$ & 47.0 & 37.0 & 88 & 73.3 & 26 & 21.7 & $<0.001$ \\
\hline \multicolumn{8}{|l|}{ Depression } \\
\hline BDI-21 (observed range $0-52)(n=128)$ & 17.0 & 13.0 & 89 & 69.5 & 31 & 24.2 & $<0.001$ \\
\hline \multicolumn{8}{|l|}{ Well-being } \\
\hline WHO-5 (observed range $0-25)(n=142)$ & 12.0 & 17.0 & 103 & 72.5 & 24 & 16.8 & $<0.001$ \\
\hline
\end{tabular}

a Score decreased over time, except for WHO-5 which increased. b Score increased over time, except for WHO-5 which decreased. c Completed at both pre-intervention and end of intervention.

Principal component analysis (Table 2) was used to investigate whether MNWS-R symptoms might belong to one or several constructs: whereas Kaiser's criterion suggested a five-component structure, the scree plot led to retention of three components (eigenvalues 5.3, 1.8, and 1.3), which explained $52.6 \%$ of the total variance. The first factor was mainly contributed by 'psychological' aspects, such as craving, anxiety, depression, irritability, restlessness, and impatience. The factor scores were significantly associated with MPSS $(\rho=0.55$, $\mathrm{p}<0.001)$, WHO-5 $(\rho=-0.67, \mathrm{p}<0.001)$, STAI-S $(\rho=0.62, p<0.001)$, and BDI-21 $(\rho=0.53, p<0.001)$. The second factor included difficulty concentrating, sleep problems, constipation, dizziness, and hot flushes or shivers. The factor scores similarly correlated with MPSS $(\rho=0.23, p=0.005)$, WHO- $5(\rho=-0.38, p<0.001)$, STAI-S $(\rho=0.52, p<0.001)$, and BDI-21 $(\rho=0.43, p<0.001)$. The third factor included increased appetite, coughing, nausea, and sore throat. The factor scores correlated with STAI-S $(\rho=0.20, p=0.03)$ and BDI-21 $(\rho=0.27, p=0.002)$, but not MPSS $(\rho=0.05, p=0.55)$ and WHO-5 $(\rho=-$ $0.09, \mathrm{p}=0.31)$.

\section{Changes between pre- and post-intervention (at 26-hour) assessments}

As indicated in Table $3 \mathrm{a}, 13$ of 16 items in the MNWS-R significantly improved after the intervention, including the following MNWS basic elements: irritability, anxiety, depressed mood, difficulty concentrating, restlessness, and craving. The only symptoms that did not change significantly were appetite, nausea, and sore throat. Anxiety (STAI-S), 
depression (BDI-21), well-being (WHO-5), and craving (MPSS) similarly improved between preintervention and 26-hour assessments (sign tests, all $\mathrm{p}<0.001)$.

Further inspection of changes (improvement or aggravation) according to NRT had very limited statistical power to detect differences in the small no-NRT subgroup, because most subjects $(86.8 \%)$ used some form of NRT. As shown in Table 3b, most items in the NRT subgroup improved significantly more than worsened. For the no-NRT subgroup, improvement was detected for anger and anxiety in the MNWS-R items but not for craving in the MNWS-R or MPSS scores. However, $48.1 \%$ with NRT improved compared with $31.6 \%$ without, and
$18.8 \%$ worsened with NRT compared with $36.8 \%$ without.

\section{Outcomes of 26-hour smoking abstinence} according to changes in withdrawal symptoms

In Table 4, the proportions of successful or unsuccessful 26-hour abstinence are compared for participants whose withdrawal symptoms worsened or improved during the intervention. The success rate was significantly lower among participants who reported worsening on the MNWS-R item 'craving' than among those who reported improving or remaining stable $(37.0 \%$ vs $66.0 \%$, Fisher's exact test $\mathrm{p}=0.008$ ). Among participants who reported increased coughing, $80.8 \%$ were successful, compared to $54.2 \%$

Table 3b. Withdrawal symptoms before the intervention and after 26-hour abstinence trial according to NRT $(\mathrm{N}=174)$

\begin{tabular}{|c|c|c|c|c|c|c|c|c|c|c|}
\hline & \multicolumn{4}{|c|}{ Improved $^{\mathrm{a}}$} & \multicolumn{4}{|c|}{ Worsenedlb } & \multicolumn{2}{|c|}{ Sign test ${ }^{d}$} \\
\hline & NRT & No NRT & NRT & No NRT & NRT & Vo NRT & VRT & No NRT & NRT & No NRT \\
\hline & $n^{c}$ & $\%$ & $n^{c}$ & $\%$ & $n^{c}$ & $\%$ & $n^{c}$ & $\%$ & $p$ & $p$ \\
\hline \multicolumn{11}{|l|}{ Withdrawal symptoms (MNWS-R) (range 0-4) } \\
\hline Angry, irritable, frustrated $(n=153)^{c}$ & 55 & 41.1 & 10 & 52.6 & 23 & 17.2 & 2 & 10.5 & $<0.001$ & 0.04 \\
\hline Anxious, nervous $(n=153)$ & 86 & 64.2 & 10 & 52.6 & 11 & 8.2 & 1 & 5.3 & $<0.001$ & 0.01 \\
\hline Depressed mood, sad $(n=151)$ & 70 & 53.0 & 6 & 31.6 & 20 & 15.2 & 3 & 15.8 & $<0.001$ & 0.51 \\
\hline Desire or craving to smoke $(n=152)$ & 64 & 48.1 & 6 & 31.6 & 25 & 18.8 & 7 & 36.8 & $<0.001$ & 1.0 \\
\hline Difficulty concentrating $(n=153)$ & 72 & 53.7 & 5 & 26.3 & 20 & 14.9 & 4 & 21.1 & $<0.001$ & 1.0 \\
\hline $\begin{array}{l}\text { Increased appetite, hungry, weight gain } \\
(n=151)\end{array}$ & 43 & 32.6 & 6 & 31.6 & 47 & 35.6 & 6 & 31.6 & 1.0 & 1.0 \\
\hline $\begin{array}{l}\text { Insomnia, sleep problems, awakening at } \\
\text { night }(n=153)\end{array}$ & 62 & 46.3 & 5 & 26.3 & 28 & 20.9 & 1 & 5.3 & 0.001 & 0.22 \\
\hline Restless ( $n=153)$ & 68 & 50.8 & 4 & 21.1 & 25 & 18.7 & 6 & 31.6 & $<0.001$ & 0.75 \\
\hline Impatient ( $n=153)$ & 65 & 48.5 & 4 & 21.1 & 33 & 24.6 & 4 & 21.1 & 0.002 & 1.0 \\
\hline Constipation $(n=163)$ & 25 & 18.8 & 2 & 10.5 & 19 & 14.3 & 1 & 5.3 & 0.45 & 1.0 \\
\hline Dizziness $(n=153)$ & 52 & 38.8 & 4 & 21.1 & 19 & 14.2 & 4 & 21.1 & $<0.001$ & 0.22 \\
\hline Coughing $(n=152)$ & 48 & 36.1 & 5 & 26.3 & 26 & 19.6 & 2 & 10.5 & 0.02 & 0.45 \\
\hline Dreaming or nightmares $(n=152)$ & 59 & 44.4 & 5 & 26.3 & 24 & 18.1 & 3 & 15.8 & $<0.001$ & 0.73 \\
\hline Nausea $(n=153)$ & 32 & 23.9 & 3 & 15.8 & 18 & 13.4 & 4 & 21.1 & 0.07 & 1.0 \\
\hline Sore throat $(n=152)$ & 32 & 24.1 & 5 & 26.3 & 25 & 18.8 & 2 & 10.5 & 0.43 & 0.45 \\
\hline Hot flushes, shivers $(n=152)$ & 52 & 39.1 & 5 & 26.3 & 22 & 16.5 & 0 & 0 & 0.001 & 0.06 \\
\hline \multicolumn{11}{|l|}{ Craving } \\
\hline MPSS (observed range $0-10)(n=157)$ & 79 & 57.2 & 8 & 47.4 & 26 & 18.8 & 6 & 31.6 & $<0.001$ & 0.61 \\
\hline \multicolumn{11}{|l|}{ Anxiety } \\
\hline STAI-S (observed range $20-77)(n=120)$ & 79 & 73.8 & 9 & 69.2 & 23 & 21.5 & 3 & 23.1 & $<0.001$ & 0.15 \\
\hline \multicolumn{11}{|l|}{ Depression } \\
\hline BDI-21 (observed range $0-52)(n=128)$ & 80 & 70.2 & 9 & 64.3 & 28 & 24.6 & 3 & 21.4 & $<0.001$ & 0.15 \\
\hline \multicolumn{11}{|l|}{ Well-being } \\
\hline WHO-5 (observed range $0-25)(n=142)$ & 93 & 73.8 & 10 & 62.5 & 21 & 16.7 & 3 & 18.8 & $<0.001$ & 0.09 \\
\hline
\end{tabular}

a Score decreased over time, except for WHO-5 which increased. b Score increased over time, except for WHO-5 which decreased. c Completed at both pre-intervention and end of intervention. d Sign test, binomial distribution used. 
Table 4. Comparison of patients whose symptoms worsened or improved during the intervention with respect to successful 26-hour abstinence $(\mathrm{N}=174)$

\begin{tabular}{|c|c|c|c|c|c|c|c|c|c|}
\hline & \multicolumn{4}{|c|}{$\begin{array}{l}\text { Symptoms worsened during } \\
26 \text { h abstinence }\end{array}$} & \multicolumn{4}{|c|}{$\begin{array}{l}\text { Symptoms were stable or } \\
\text { improved during } 26 \text { h abstinence }\end{array}$} & \multirow{3}{*}{$\begin{array}{c}\text { Fisher's } \\
\text { test } \\
\text { p }\end{array}$} \\
\hline & \multicolumn{2}{|c|}{$\begin{array}{l}\text { Successful } \\
\text { abstinence }\end{array}$} & \multicolumn{2}{|c|}{$\begin{array}{l}\text { Unsuccessful } \\
\text { abstinence }\end{array}$} & \multicolumn{2}{|c|}{$\begin{array}{l}\text { Successfinl } \\
\text { abstinence }\end{array}$} & \multicolumn{2}{|c|}{$\begin{array}{l}\text { Unsuccessful } \\
\text { abstinence }\end{array}$} & \\
\hline & n & $\%$ & n & $\%$ & n & $\%$ & $n$ & $\%$ & \\
\hline \multicolumn{10}{|l|}{ Withdrawal symptoms (MNWS-R) } \\
\hline Angry, irritable, frustrated $(n=134)$ & 9 & 42.9 & 12 & 57.1 & 71 & 62.8 & 42 & 37.2 & 0.10 \\
\hline Anxious, nervous ( $n=134)$ & 4 & 44.4 & 5 & 55.6 & 76 & 60.8 & 49 & 39.2 & 0.48 \\
\hline Depressed mood, sad $(n=132)$ & 10 & 47.6 & 11 & 52.4 & 69 & 62.2 & 42 & 37.8 & 0.23 \\
\hline Desire or craving to smoke $(n=133)$ & 10 & 37.0 & 17 & 63.0 & 70 & 66.0 & 36 & 34.0 & 0.008 \\
\hline Difficulty concentrating $(n=134)$ & 10 & 52.6 & 9 & 47.4 & 70 & 60.9 & 45 & 39.1 & 0.62 \\
\hline Increased appetite, hungry, weight gain $(n=132)$ & 26 & 60.5 & 17 & 39.5 & 54 & 60.7 & 35 & 39.3 & 1.00 \\
\hline Insomnia, sleep problems, awakening at night $(n=134)$ & 13 & 52.0 & 12 & 48.0 & 67 & 61.5 & 42 & 38.5 & 0.50 \\
\hline Restless $(n=134)$ & 14 & 48.3 & 15 & 51.7 & 66 & 62.9 & 39 & 37.1 & 0.20 \\
\hline Impatient $(n=134)$ & 15 & 48.4 & 16 & 51.6 & 65 & 63.1 & 38 & 36.9 & 0.15 \\
\hline Constipation $(n=133)$ & 12 & 66.7 & 6 & 33.3 & 68 & 59.1 & 47 & 40.9 & 0.61 \\
\hline Dizziness $(n=134)$ & 10 & 55.6 & 8 & 44.4 & 70 & 60.3 & 46 & 39.7 & 0.80 \\
\hline Coughing $(n=133)$ & 21 & 80.8 & 5 & 19.2 & 58 & 54.2 & 49 & 45.8 & 0.02 \\
\hline Dreaming or nightmares $(n=133)$ & 17 & 70.8 & 7 & 29.2 & 63 & 57.8 & 46 & 42.2 & 0.26 \\
\hline Nausea $(n=134)$ & 10 & 50.0 & 10 & 50.0 & 70 & 61.4 & 44 & 38.6 & 0.46 \\
\hline Sore throat $(n=133)$ & 15 & 62.5 & 9 & 37.5 & 64 & 58.7 & 45 & 41.3 & 0.82 \\
\hline Hot flushes, shivers $(n=133)$ & 12 & 60.0 & 8 & 40.0 & 68 & 60.2 & 45 & 39.8 & 1.00 \\
\hline \multicolumn{10}{|l|}{ Craving } \\
\hline MPSS $(n=138)$ & 12 & 41.4 & 17 & 58.6 & 70 & 64.2 & 39 & 35.8 & 0.03 \\
\hline \multicolumn{10}{|l|}{ Anxiety } \\
\hline STAI-S $(n=113)$ & 11 & 45.8 & 13 & 54.2 & 60 & 67.4 & 29 & 32.6 & 0.06 \\
\hline \multicolumn{10}{|l|}{ Depression } \\
\hline BDI-21 $(n=120)$ & 12 & 41.4 & 17 & 58.6 & 62 & 83.8 & 29 & 31.9 & 0.02 \\
\hline \multicolumn{10}{|l|}{ Well-being } \\
\hline WHO-5 ( $n=133)$ & 21 & 55.3 & 17 & 44.7 & 59 & 62.1 & 36 & 37.9 & 0.56 \\
\hline
\end{tabular}

of those without increased coughing $(\mathrm{p}=0.02)$. For all other MNWS-R items, improvement or aggravation was not significantly associated with the outcome of the 26-hour abstinence intervention. The proportion of success was also significantly lower with worsening on the MPSS craving subscale $(41.4 \%$ vs $64.2 \%$, $\mathrm{p}=0.03$ ) and the BDI-21 (increasing depression score) $(41.4 \%$ vs $83.8 \%, \mathrm{p}=0.02)$.

\section{DISCUSSION}

In the present sample of patients with severe mental health disorders, the prevalence of moderate to severe anxiety (STAI-S) and depression (BDI21 ) was $>40 \%$ at pre-intervention. 'Withdrawal symptoms' (MNWS-R) were already observable at pre-intervention, with median scores of 2 for anxiety, depression, craving, difficulty concentrating, restlessness, and impatience. This observation may raise the question of whether withdrawal symptoms might already have been present during continued smoking. In any case, the results confirm the methodological difficulties in studying withdrawal effects in psychiatric patients, because these symptoms are also frequent in psychiatric conditions. During the 26-hour abstinence trial, craving appeared to be a more prominent symptom rated higher than other symptoms on the MNWS-R scale, also among patients with confounding symptoms due to mental illness. It was a severe symptom in $28.8 \%$ of participants. This finding is consistent with observations showing 
that abstinence effects are largest for craving ${ }^{40}$. A factorial analysis of the MNWS-R showed that craving belonged to a factor that included anxiety, depression, irritability, restlessness, and impatience, thus underlining the difficulty in disentangling it from other symptoms. Significant correlations of all three withdrawal components with anxiety and depression scores further confirmed the difficulty in interpreting such factors. However, conceptually, craving was the only item specifically related to smoking, thus suggesting that although it is associated with other aspects of withdrawal, it remains central in evaluating smoking abstinence. Withdrawal seems related to a non-specific reaction to the stress of abstinence, whereas craving could be considered the 'tip of the iceberg' in the withdrawal syndrome.

Unexpectedly, analysis of changes before and after intervention showed that patients' traditional withdrawal symptoms clearly improved during abstinence, in contrast with descriptions of increased discomfort after smoking cessation. For most withdrawal symptoms, including craving, as well as for psychiatric symptoms (anxiety, depression) and well-being, improvement between pre- and postintervention was significantly more common than aggravation.

Understanding the observed improvement of withdrawal symptoms after abstinence is challenging, and limitations to study this topic include the following:

- This intervention includes smokers not searching counseling support for cessation, in contrast to most studies of smoking-cessation. Subjects seeking assistance in cessation might have more acute withdrawal symptoms.

- The diversity in individual smoking profiles was high, because the present intervention was designed to include all smokers according to a broad definition. Although mean tobacco use was important, $32.4 \%$ of participants smoked $\leq 10$ cigarettes per day. Hence, one-third of smokers probably did not experience important tobacco withdrawal symptoms and related discomfort.

- The sample also included one-third of heavy smokers $(33.6 \%$ smoked $>20$ cigarettes per day) for whom high anticipatory anxiety at preintervention might have contributed to the global decrease in negative affect during the intervention.
Patients with mental health disorders might also have higher anxiety or be less able to manage negative affect. We observed that heavy smokers or those smoking for decades sometimes showed substantial anticipatory uncertainty about their ability to not smoke for 26 hours. The literature has reported anticipatory and psychological reactions to cessation and, for some smokers, increased negative affect even before cessation ${ }^{41}$, and unfounded fears about having a strong desire to smoke after cessation have been described in non-psychiatric samples ${ }^{42}$.

- All subjects in our sample received psychiatric treatment including pharmacotherapy, which may have blunted the increase in withdrawal symptoms during smoking abstinence. Improvements associated with mental health treatment may also have occurred between pre- and post-intervention; however, such effects probably remained marginal, given the short period.

- Finally, components of the intervention itself should be mentioned: having a busy and pleasant schedule, being out of one's usual environment, the need for increased attentional resources and being distracted from everyday functioning, engaging in light physical exercise (thermal baths), receiving group support from other participants and professionals, and experiencing the positive psychological effects of a successful intervention might have contributed to decreased withdrawal symptoms.

Our study results provide deeper understanding of the relationship between short-term 26-hour smoking abstinence success and withdrawal symptoms. Among all the MNWS-R items, only craving and coughing, as well as craving on the MPSS subscale and depression on the clinical depression scale (BDI-21), were significantly associated with successful abstinence.

Craving evolved differently between successful and unsuccessful abstinence, clearly decreasing during the intervention for abstainers (more than half the sample), whereas it remained high in those who relapsed. The significant association between stronger craving and a failure to maintain temporary abstinence confirms that craving can be considered a risk factor for smoking relapse after cessation, but this association would mainly apply to the subgroup 
with unsuccessful cessation attempts. Intense craving during a quit attempt might be a sign of unsuccessful abstinence. The finding that successful abstainers showed lower craving during abstinence indicates that increased craving is not necessarily associated with interruption of nicotine intake from cigarettes. Such considerations were discussed more than 30 years ago, as lighter craving was observed in subjects totally abstaining from smoking than in those continuing smoking ${ }^{43}$. Other studies confirmed that post-cessation craving is a better indicator of successful abstinence than pre-cessation craving ${ }^{44}$, and that exacerbation of symptoms of depression is more strongly associated with unsuccessful cessation attempts than successful abstinence ${ }^{45,46}$. Differences in craving between successful and unsuccessful abstainers shortly after cessation has further been described $^{41}$, thus indicating that smokers' paths diverge more after than before a cessation attempt. In particular, craving is more tightly linked with reports of recent smoking during a cessation attempt than any other symptom. Thus, to predict success, craving should be assessed post-cessation. Craving is interesting as relapse indicator only in the case of abstinence; consequently, craving may be a useful indicator of cessation success during abstinence, but it may be conceptualized as a core symptom of smoking forming part of a 'chronic withdrawal cycle $^{345}$.

In our data, other significant differences between successful and unsuccessful 26-hour smoking abstinence trials involved 'coughing' in the MNWS-R, which was more pronounced for successful abstainers. Coughing is a common and normal reaction after smoking $\operatorname{cessation}^{47}$, owing to the reactivation of airway clearance by the mucociliary system. Cigarette smokers have diminished cough-reflex sensitivity compared to non-smokers, eventually related with a centrally mediated antitussive effect of nicotine ${ }^{48}$, and intensity of smoking is known to be associated with impairment of nasal mucociliary clearance. Smoking cessation rapidly induces improvements in mucociliary clearance ${ }^{49}$ and reverses inhibition of cough-reflex sensitivity ${ }^{50}$. Our data suggest that this could occur very rapidly, already after 26 hours of smoking abstinence, for participants who did not smoke.
Our study also confirmed that depression, assessed on a validated clinical scale, improved more for abstainers, as evaluated during a short-term period. In the longer term, improvement in mental health conditions upon smoking cessation relies on a growing body of research indicating positive experiences with psychiatric patients stopping smoking and improvements in psychological functioning for quitters even in groups with severe psychiatric conditions ${ }^{45}$. The literature indicates that lower stress after smoking cessation is a robust and empirical phenomenon ${ }^{51}$ and that abstinence is not associated with increased affective distress, even in smokers with depressive disorders ${ }^{45}$. Our results pertain to only a short 26-hour abstinence trial, but they clearly illustrate that even severely dependent smokers, in a favorable context with NRT, mostly did not present with severe withdrawal effects or increased negative affect when smoking was interrupted. Most subjects used NRT, and our results and positive outcomes were thus influenced by this treatment.

The long duration of the recruitment period ( 7 years) resulted from an adaptation of the frequency of 'Day off' interventions to the organizational possibilities of the Department of Psychiatry and the number of patients ready to commit to this intensive program, because most smokers were in pre-contemplation stages. However, no major changes that might have influenced smoking, such as hospital regulations (an indoor smoking ban) or forms of NRT used, were observed during this period.

\section{Limitations}

Several limitations apply to this study. First, a control group was not included. Comparison with controls not attending the 'Day off' intervention as well as comparison of a group participating but not undergoing abstinence would be necessary to further study the effects of smoking withdrawal. Further, more intense monitoring, such as experience sample measurement, would provide additional information about the short-term evolution of craving. Another limitation is the short 26-hour time frame of analysis, because withdrawal symptoms change over time, and assessment after longer periods is necessary to study the effects after smoking cessation not only after 
temporary abstinence ${ }^{7,52}$. Tobacco withdrawal was observed to peak at 1 month, and short- and longterm symptoms differed ${ }^{6}$. The sample was insufficient for analyses of NRT use to elucidate characteristics of no-NRT users, because of the small number of subjects in this category. Interpretation was further limited by the absence of correction for confounding factors. For example, NRT use may be related to the strength of dependency, but sample size limits do not enable in depth investigation.

Finally we emphasize the complexity of smoking patterns, encompassing large interindividual differences leading to different withdrawal symptom patterns and abstinence attempt outcomes ${ }^{53}$. Most smokers report typical levels of withdrawal symptoms on cessation day, and more than one-third report extreme craving or negative affect or hunger after initial abstinence. The existence of different subgroups of smokers differing in tobacco-related, negative affect, sociodemographic, or outcome variables ${ }^{53}$ requires further research to clarify the associations between individual withdrawal profiles and psychopathology.

\section{CONCLUSIONS}

Our results confirm the difficulty of assessing tobacco withdrawal in persons with mental health concerns. Interestingly, contrary to our expectations, short-term smoking related withdrawal symptoms did not increase but instead decreased in most psychiatric patients, who experienced abstinence in a stimulating and professional supportive context. Our data moderate the common assumption or myth that smoking abstinence leads to increased craving, particularly when NRT is available.

Craving, however, remained an interesting symptom to assess. It was the most prevalent withdrawal symptom, decreased more frequently among abstinent subjects, thus suggesting that craving might be a core symptom of smoking itself and may be an interesting predictor of cessation success, but only when measured during a cessation attempt.

This study highlighted that even patients with mental disorders can participate, and experience many benefits, in a motivational enhancement program based on a 26-hour tobacco abstinence experience.

\section{REFERENCES}

1. Moore RA, Aubin HJ. Do placebo response rates from cessation trials inform on strength of addictions? Int J Environ Res Public Health. 2012;9(1):192-211. doi:10.3390/ijerph9010192

2. Morissette A, Ouellet-Plamondon C, Jutras-Aswad D. [Craving as a core symptom in substance use disorders: epidemiology, neurobiological substrates and clinical relevance]. Sante Ment Que. 2014;39(2):21-37. doi:10.7202/1027830ar

3. Skinner MD, Aubin HJ. Craving's place in addiction theory: contributions of the major models. Neurosci Biobehav Rev. 2010;34(4):606-623. doi:10.1016/j.neubiorev.2009.11.024

4. Tiffany ST, Wray JM. The clinical significance of drug craving. Ann N Y Acad Sci. 2012;1248(1):1-17. doi:10.1111/j.1749-6632.2011.06298.x

5. Piper ME. Withdrawal: Expanding a Key Addiction Construct. Nicotine Tob Res. 2015;17(12):1405-1415. doi:10.1093/ntr/ntv048

6. Gritz ER, Carr CR, Marcus AC. The tobacco withdrawal syndrome in unaided quitters. Br J Addict. 1991;86(1):5769. doi:10.1111/j.1360-0443.1991.tb02629.x

7. Hughes JR, Gust SW, Skoog K, Keenan RM, Fenwick JW. Symptoms of tobacco withdrawal. A replication and extension. Arch Gen Psychiatry. 1991;48(1):52-59.

8. American Psychiatric Association. Diagnostic and statistical manual of mental disorders. 4th ed. Washington, DC: APA; 1994. doi:10.1017/s0033291700035765

9. Toll BA, O’Malley SS, McKee SA, Salovey P, KrishnanSarin S. Confirmatory factor analysis of the Minnesota Nicotine Withdrawal Scale. Psychol Addict Behav. 2007;21(2):216-225. doi:10.1037/0893-164X.21.2.216

10. Baker TB, Piper ME, Schlam TR, Cook J, Smith S, Loh WY, Bolt D. Are tobacco dependence and withdrawal related amongst heavy smokers? Relevance to conceptualizations of dependence. J Abnorm Psychol. 2012;121(4):909-921. doi:10.1037/a0027889

11. West R, Ussher M, Evans M, Rashid M. Assessing DSMIV nicotine withdrawal symptoms: a comparison and evaluation of five different scales. Psychopharmacology (Berl). 2006;184(3-4):619-627. doi:10.1007/s00213005-0216-z

12. Jackson JG, Diaz FJ, Lopez L, de Leon J. A combined analysis of worldwide studies demonstrates an association between bipolar disorder and tobacco smoking behaviors in adults. Bipolar Disord. 2015;17(6):575-597. doi:10.1111/bdi.12319

13. Steinberg ML, Williams JM, Li Y. Poor Mental Health and Reduced Decline in Smoking Prevalence. Am J Prev Med. 2015;49(3):362-369. doi:10.1016/j.amepre.2015.01.016

14. Cather C, Pachas GN, Cieslak KM, Evins AE. Achieving Smoking Cessation in Individuals with Schizophrenia: Special Considerations. CNS Drugs. 2017;31(6):471-481. doi:10.1007/s40263-017-0438-8 
15. Vermeulen J, Schirmbeck F, Blankers M, van Tricht M, van den Brink W, de Haan L. Smoking, symptoms, and quality of life in patients with psychosis, siblings, and healthy controls: a prospective, longitudinal cohort study. Lancet Psychiatry. 2019;6(1):25-34. doi:S2215-0366(18)30424-3

16. Cook BL, Wayne GF, Kafali EN, Liu Z, Shu C, Flores M. Trends in smoking among adults with mental illness and association between mental health treatment and smoking cessation. JAMA. 2014;311(2):172-182. doi:10.1001/jama.2013.284985

17. Keizer I, Gex-Fabry M, Bruegger A, Croquette P, Khan AN. Staff representations and tobacco-related practices in a psychiatric hospital with an indoor smoking ban. Int J Ment Health Nurs. 2014;23(2):171-182. doi:10.1111/inm.12030

18. Prochaska JJ. Smoking and mental illness--breaking the link. N Engl J Med. 2011;365(3):196-198. doi:10.1056/NEJMp1105248

19. Hitsman B, Moss TG, Montoya ID, George TP. Treatment of tobacco dependence in mental health and addictive disorders. Can J Psychiatry. 2009;54(6):368-378. doi:10.1177/070674370905400604

20. Burns A, Webb M, Stynes G et al. Implementation of a Quit Smoking Programme in Community Adult Mental Health Services-A Qualitative Study. Front Psychiatry. 2018;9:670. doi:10.3389/fpsyt.2018.00670

21. Taylor G, McNeill A, Girling A, Farley A, Lindson-Hawley $\mathrm{N}$, Aveyard P. Change in mental health after smoking cessation: systematic review and meta-analysis. BMJ. 2014;348:g151. doi:10.1136/bmj.g1151

22. Manzella F, Maloney SE, Taylor GT. Smoking in schizophrenic patients: A critique of the self-medication hypothesis. World J Psychiatry. 2015;5(1):35-46. doi:10.5498/wjp.v5.i1.35

23. Soyster P, Anzai NE, Fromont SC, Prochaska JJ. Correlates of nicotine withdrawal severity in smokers during a smoke-free psychiatric hospitalization. Prev Med. 2016;92:176-182. doi:10.1016/j.ypmed.2016.01.026

24. Gray KM, Baker NL, Carpenter MJ, Lewis AL, Upadhyaya HP. Attention-deficit/hyperactivity disorder confounds nicotine withdrawal self-report in adolescent smokers. Am J Addict. 2010;19(4):325-331. doi:10.1111/j.1521-0391.2010.00048.x

25. Pomerleau CS, Downey KK, Snedecor SM, Mehringer AM, Marks JL, Pomerleau OF. Smoking patterns and abstinence effects in smokers with no ADHD, childhood ADHD, and adult ADHD symptomatology. Addict Behav. 2003;28(6):1149-1157. doi:10.1016/S0306-4603(02)00223-x

26. Hughes JR, Hatsukami D. Signs and symptoms of tobacco withdrawal. Arch Gen Psychiatry. 1986;43(3):289-294.

27. Hughes JR. Background on the Minnesota Tobacco Withdrawal Scale - Revised (MTWS-R). http:// contentmanager.med.uvm.edu/docs/background/ behavior-and-health-documents/background. pdf?sfvrsn=2. Published March 1, 2017. Accessed February 27, 2018.

28. Parschau L, Fleig L, Warner LM, et al. Positive Exercise Experience Facilitates Behavior Change via SelfEfficacy. Health Educ Behav. 2014;41(4):414-422. doi:10.1177/1090198114529132

29. Keizer I, Gex-Fabry M, Croquette P, Khan AN. A short motivational program based on temporary smoking abstinence: Towards increased self-efficacy to quit in psychiatric inpatients. J Addict Res Ther. 2016;7(4). doi:10.4172/2155-6105.1000289

30. Etter JF. [Minnesosta (MNWS-R)]. http://www.stoptabac.ch/fr/WDR/. Accessed December 8, 2014.

31. West R, Hajek P. Evaluation of the mood and physical symptoms scale (MPSS) to assess cigarette withdrawal. Psychopharmacology (Berl). 2004;177(1-2):195-199. doi:10.1007/s00213-004-1923-6

32. Etter JF, Vu Duc T, Perneger TV. Validity of the Fagerström test for nicotine dependence and of the Heaviness of Smoking Index among relatively light smokers. Addiction. 1999;94(2):269-281. doi:10.1046/j.1360-0443.1999.94226910.x

33. Kozlowski LT, Porter CQ, Orleans CT, Pope MA, Heatherton T. Predicting smoking cessation with selfreported measures of nicotine dependence: FTQ, FTND, and HSI. Drug Alcohol Depend. 1994;34(3):211-216. doi:10.1016/0376-8716(94)90158-9

34. Biener L, \& Abrams DB. The Contemplation Ladder: validation of a measure of readiness to consider smoking cessation. Health Psychol. 1991, 10(5), 360-365.

35. Spielberger CD. State-trait Anxiety Inventory: A comprehensive Bibliography. Palo Alto, CA: Consulting Psychologists Press; 1983.

36. Beck AT, Epstein N, Brown G, Steer RA. An inventory for measuring clinical anxiety: psychometric properties. J Consult Clin Psychol. 1988;56(6):893-897. doi:10.1037/0022-006X.56.6.893

37. World Health Organization. Indice (en 5 points) de bienêtre de l'OMS (1999). Hillerod, Denmark: Psychiatric Research Unit, WHO Collaborating Center for Mental Health, Frederiksborg General Hospital; 1999. https:// www.psykiatri-regionh.dk/who-5/Documents/WHO5_ French.pdf. Accessed March 8, 2011.

38. Bech P, Gudex C, Johansen KS. The WHO (Ten) WellBeing Index: validation in diabetes. Psychother Psychosom. 1996;65(4):183-190. doi:10.1159/000289073

39. Newnham EA, Hooke GR, Page AC. Monitoring treatment response and outcomes using the World Health Organization's Wellbeing Index in psychiatric care. J Affect Disord. 2010;122(1-2):133-138. doi:10.1016/j.jad.2009.06.005

40. Leventhal AM, Waters AJ, Moolchan ET, Heishman SJ, Pickworth WB. A quantitative analysis of subjective, cognitive, and physiological manifestations 
of the acute tobacco abstinence syndrome. Addict Behav. 2010;35(12):1120-1130. doi:10.1016/j. addbeh.2010.08.007

41. McCarthy DE, Piasecki TM, Fiore MC, Baker TB. Life before and after quitting smoking: an electronic diary study. J Abnorm Psychol. 2006;115(3):454-466. doi:10.1037/0021-843X.115.3.454

42. Boehm G, Schroeder Y, Schoberberger R. Inpatient smoking cessation therapy: truth or dare? Wien Klin Wochenschr. 2015;127(19-20):786-791. doi:10.1007/s00508-015-0820-9

43. Shiffman SM, Jarvik ME. Smoking withdrawal symptoms in two weeks of abstinence. Psychopharmacology (Berl). 1976;50(1):35-39.

44. Wray JM, Gass JC, Tiffany ST. A systematic review of the relationships between craving and smoking cessation. Nicotine Tob Res. 2013;15(7):1167-1182. doi:10.1093/ntr/nts268

45. Berlin I, Chen H, Covey LS. Depressive mood, suicide ideation and anxiety in smokers who do and smokers who do not manage to stop smoking after a target quit day. Addiction. 2010;105(12):2209-2216. doi:10.1111/j.1360-0443.2010.03109.x

46. McClave AK, Dube SR, Strine TW, Kroenke K, Caraballo RS, Mokdad AH. Associations between smoking cessation and anxiety and depression among U.S. adults. Addict Behav. 2009;34(6-7):491-497. doi:10.1016/j.addbeh.2009.01.005

47. Cummings KM, Giovino G, Jaen CR, Emrich LJ. Reports of smoking withdrawal symptoms over a 21 day period of abstinence. Addict Behav. 1985;10(4):373-381. doi:10.1016/0306-4603(85)90034-6

48. Dicpinigaitis PV. Effect of tobacco and electronic cigarette use on cough reflex sensitivity. Pulm Pharmacol Ther. 2017;47:45-48. doi:10.1016/j.pupt.2017.01.013

49. Utiyama DM, Yoshida CT, Goto DM, et al. The effects of smoking and smoking cessation on nasal mucociliary clearance, mucus properties and inflammation. Clinics (Sao Paulo). 2016;71(6):344-350. doi:10.6061/ clinics/2016(06)10

50. Sitkauskiene B, Stravinskaite K, Sakalauskas R, Dicpinigaitis PV. Changes in cough reflex sensitivity after cessation and resumption of cigarette smoking. Pulm Pharmacol Ther. 2007;20(3):240-243. doi:10.1016/j.pupt.2006.08.005

51. Mathew AR, Robinson JD, Norton PJ, Cinciripini PM, Brown RA, Blalock JA. Affective trajectories before and after a quit attempt among smokers with current depressive disorders. Nicotine Tob Res. 2013;15(11):1807-1815. doi:10.1093/ntr/ntt036

52. Piper ME, Vasilenko SA, Cook JW, Lanza ST. What a difference a day makes: differences in initial abstinence response during a smoking cessation attempt. Addiction. 2017;112(2):330-339. doi:10.1111/add.13613

53. Parrott AC, Murphy RS. Explaining the stress- inducing effects of nicotine to cigarette smokers. Hum Psychopharmacol. 2012;27(2):150-155. doi:10.1002/hup.1247

\section{ACKNOWLEDGEMENTS}

We thank the following heads of services for their collaboration in the intervention: Jean-Pierre Bachetta, Javier Bartolomei, Logos Curtis, Philippe Huguelet, Othman Sentissi, and the heads of the Department of Mental Health and Psychiatry, Panteleimon Giannacopoulos and Jean-Michel Aubry for support.

We thank the tobacco specialists for their interventions in the program, in particular Corinne Wahl (Carrefour addictions/ CIPRET-Geneva), who participated in all the outpatient 'Day off' sessions and provided detailed tobacco information, and Patricia Borrero (Directorate of Nursing Services, University Hospitals of Geneva), who provided interactive tobaccoinformation sessions for the inpatient groups.

\section{CONFLICTS OF INTEREST}

The authors have completed and submitted the ICMJE Form for Disclosure of Potential Conflicts of Interest and none was reported.

\section{FUNDING}

This study received no specific funding; the authors were employed by the University Hospitals of Geneva and/or the University of Geneva. Small funds to implement the outpatient portion of the 'Day off' intervention were provided by Carrefour addictions/CIPRET-Genève, Centre d'Information et de Prévention du Tabagisme. All participating institutions are funded by the state of Geneva, and the study involved no private funds.

\section{PROVENANCE AND PEER REVIEW}

Not commissioned; externally peer reviewed. 